\title{
A rare case of low transverse vaginal septum
}

\section{Sanjay Singh*, Manash Biswas, Madhusudan Dey, Vijayalakshmi Nambula, Fatima Abbas}

Department of Obstetrics and Gynecology, Armed Forces Medical College, Pune, Maharashtra, 4110401, India

Received: 21 October 2015

Accepted: 18 November 2015

\section{*Correspondence:}

Dr. Sanjay Singh,

E-mail: drsanjaysingh@gmail.com

Copyright: (C) the author(s), publisher and licensee Medip Academy. This is an open-access article distributed under the terms of the Creative Commons Attribution Non-Commercial License, which permits unrestricted non-commercial use, distribution, and reproduction in any medium, provided the original work is properly cited.

\begin{abstract}
Transverse vaginal septum is a rare cause of genital outflow tract obstruction. It results from incomplete fusion/recanalization between the vaginal components of the mullerian ducts and the urogenital sinus. The septum varies in thickness and may be present anywhere along the vagina, although most are found in the upper and midvagina. A complete septum results in primary amenorrhoea and several short term and long term sequelae including endometriosis and infertility. We present a case of 12-year old girl who presented with intermittent, colicky abdominal pain. She had undergone low anorectoplasty at 18 months of age. She had not yet attained menarche. Secondary sexual characters were appropriately developed. The clinical examination revealed a small, blind ending lower vagina and a tender, bimanually palpable mobile mass in the suprapubic region. The ultrasound examination revealed fluid collection with internal echoes within the endometrial cavity extending into the upper vagina. The magnetic resonance imaging of pelvis confirmed the thickness (four $\mathrm{cm}$ ) and location of the transverse vaginal septum (lower one third) along with presence of hematocolpos in upper 2/3rd of the vagina, hematometra and hematosalpinx. She underwent successful excision of the septum. Amnion was used for the epithelialisation of the denuded vaginal mucosa.
\end{abstract}

Keywords: Transverse vaginal septum, Primary amenorrhoea, Menarche, Urogenital sinus, Hematocolpos

\section{INTRODUCTION}

Transverse vaginal septum is a rare developmental disorder of the vagina. Though it may occur anywhere along the vagina, it is least common at its lower third. Clinical presentation depends on whether it is complete or partial. Diagnosis of transverse vaginal septum is still a challenge during early infancy. With complete septum, the onset of symptoms usually occurs after puberty. Affected females may present with primary amenorrhoea or cyclical pelvic pain or midline suprapubic tender mass because of accumulation of blood in vagina (hematocolpos) or uterus (hematometra). Incomplete septa allow partial egress of menstrual blood and such patients usually present late with dysmenorrhea and dyspaerunia. Magnetic resonance imaging (MRI) has an indispensable role in distinguishing transverse vaginal septum from the more common imperforate hymen and agenesis or severe hypoplasia of the cervix. Here we present a rare case of low transverse vaginal septum.

\section{CASE REPORT}

A 12 years old girl presented with cyclical (typically between 25 th to 30th days of every month) colicky lower abdominal pain, present for five months. It was selfremitting after five days. She had not yet attained menarche. Her developmental milestones including secondary sexual characters were normal. She had low anorectal malformation at birth for which she had undergone low anorectoplasty at eighteen months of age. 
Her bowel and bladder habits were normal. She was thin built and averagely nourished with a body mass index of 16/sqm. Breast examination suggested Tanner stage III of the development of breast. On abdominal examination an eighteen weeks size of tender suprapubic midline lump was palpable. External genitalia were well developed with tanner stage III distribution of pubic hairs. Urethral and anal opening were normal. Instead of normal vaginal opening a vaginal dimple could be noticed. There was no bulging of the hymen (Figure 1). On rectal examination, there was bulging of upper vagina towards the rectum.

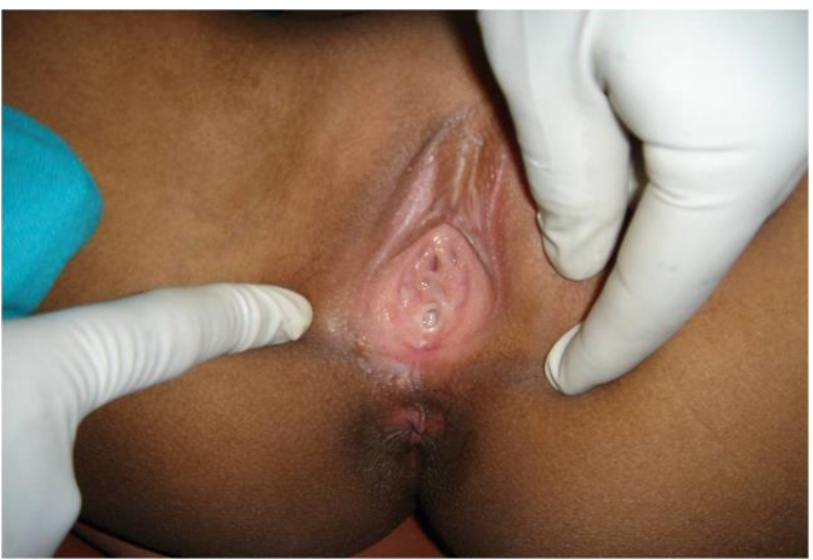

Figure 1: (Pre-op) Showing vaginal dimple in a case of transverse vaginal septum.

The ultrasound examination revealed sonolucent collection within the upper vagina extending into the endometrial cavity. Internal echoes were noted within the collection. Both ovaries were well visualized and were normal. Bilateral hydronephrosis and hydroureter were also present. Magnetic Resonance Imaging (MRI) confirmed marked collection of fluid in the upper two third of the vagina with lower end of collection stopping $2.99 \mathrm{~cm}$ proximal to external urethral orifice. The thickness of the vaginal septum was four $\mathrm{cm}$. Hematometra and hematosalpinx were also present (Figure 2). The cervix, ovaries, rectum and the bladder were normal. She was diagnosed as a case of low transverse vaginal septum and was planned for its resection.

Under USG guidance after ensuring the thickness of the septum with a needle, a small transverse incision was given over the septum and collected hemorrhagic fluid was drained (Figure 3). The septum then was excised circumferentially keeping a metal catheter in the bladder and a hegar dilator in the rectum as a guide to prevent inadvertent injury to these organs. Since large circumferential vaginal mucosa was exposed end to end anastomosis was not possible. Thus a mould covered with amnion was placed in vaginal tract to keep it patent and for the epithelialisation of the denuded vaginal mucosa (Figure 4). Labial stitches were taken to keep the mould in situ. On day four stitches were removed and redundant amnion tissue was excised. Vaginal washing with normal saline followed by insertion of the mould lubricated with estradiol cream was done on alternate days for two weeks.

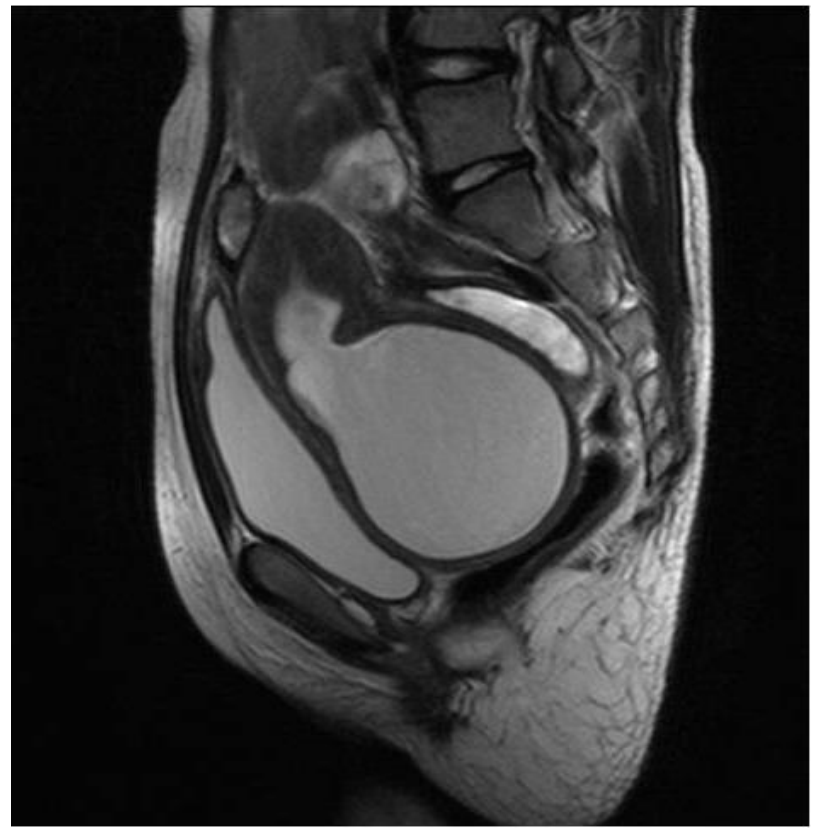

Figure 2: Magnetic resonance imaging of the pelvis in a 12-year old girl. The midline sagittal T2-weighted image shows hyper intense collection in the endometrial cavity, upper two thirds of the vagina and a thick septum separating upper two-thirds of vagina from lower $1 / 3^{\text {rd }}$.

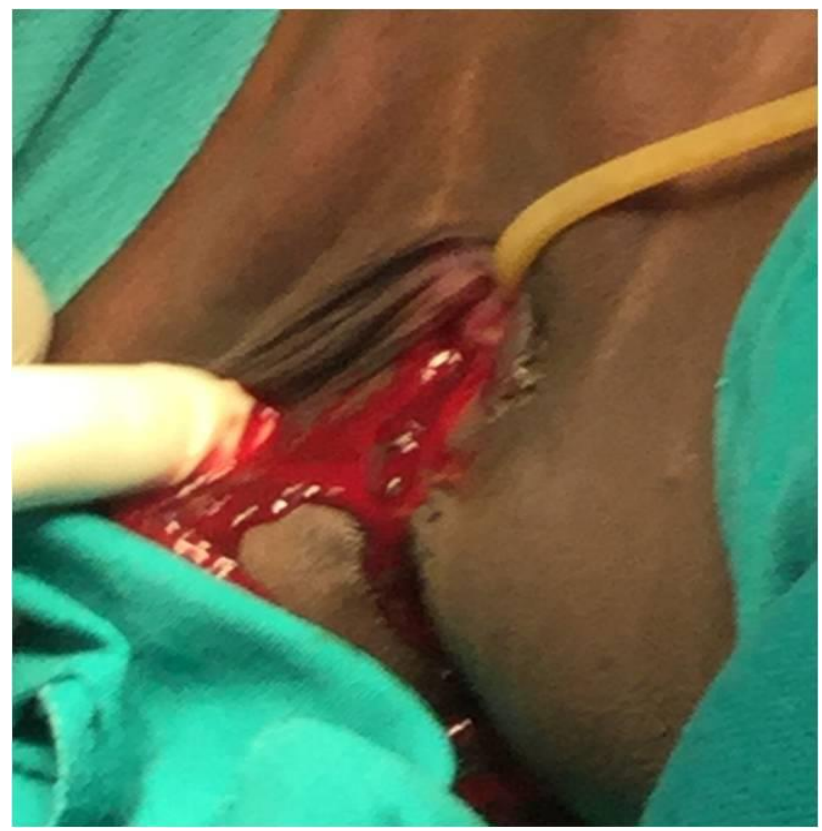

Figure 3: Drainage of collected hemorrhagic fluid after incising the transverse vaginal septum.

She is presently under follow up and started normal menstrual flow exactly after one month of the last cyclical pelvic pain. Vaginal tract is patent (Figure 5). 
She has been taught and advised for daily self dilatation with acrylic obturator for four months and then monthly, in order to keep the vaginal tract open and to prevent stenosis. Post op MRI revealed total disappearance of collection and normal uterus and vagina (Figure 6).

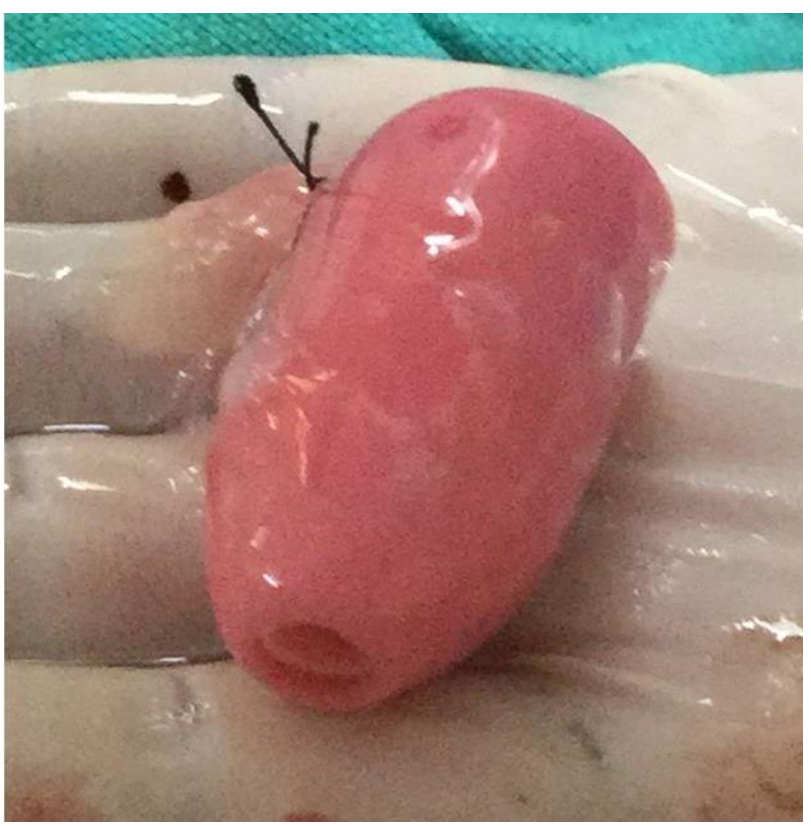

Figure 4: Vaginal mould covered with amnion.

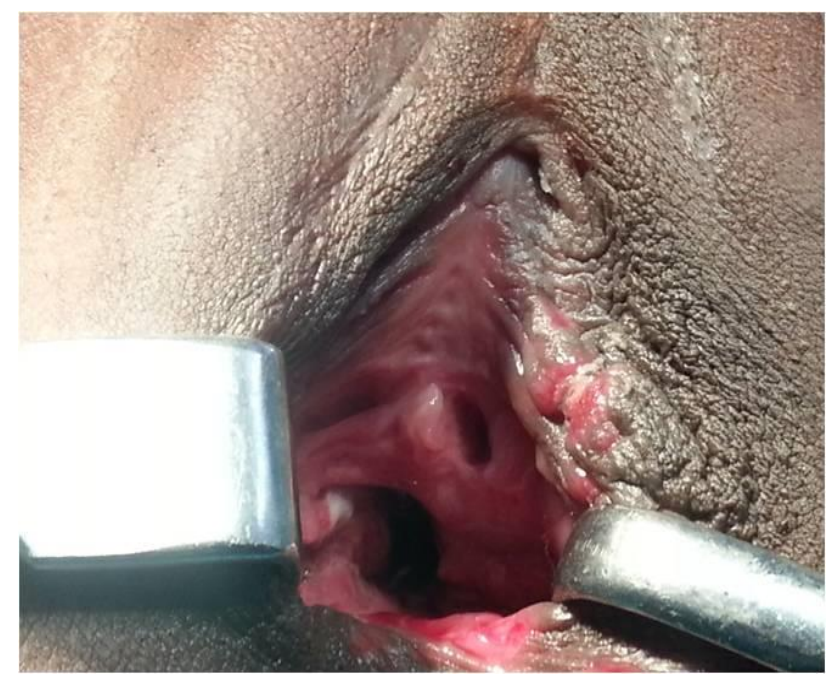

Figure 5: (Post- op) Showing patent vagina in the operated case of transverse vaginal septum.

\section{DISCUSSION}

Transverse vaginal septum is an uncommon congenital anomaly resulting in uterovaginal tract obstruction with an incidence of 1 in 70,000 females. ${ }^{1}$ There is a controversy regarding formation of the vagina. Some authors consider whole vagina to be developed from the endodermal sinovaginal bulbs while the others believe that the upper portion is derived from the paramesonehric

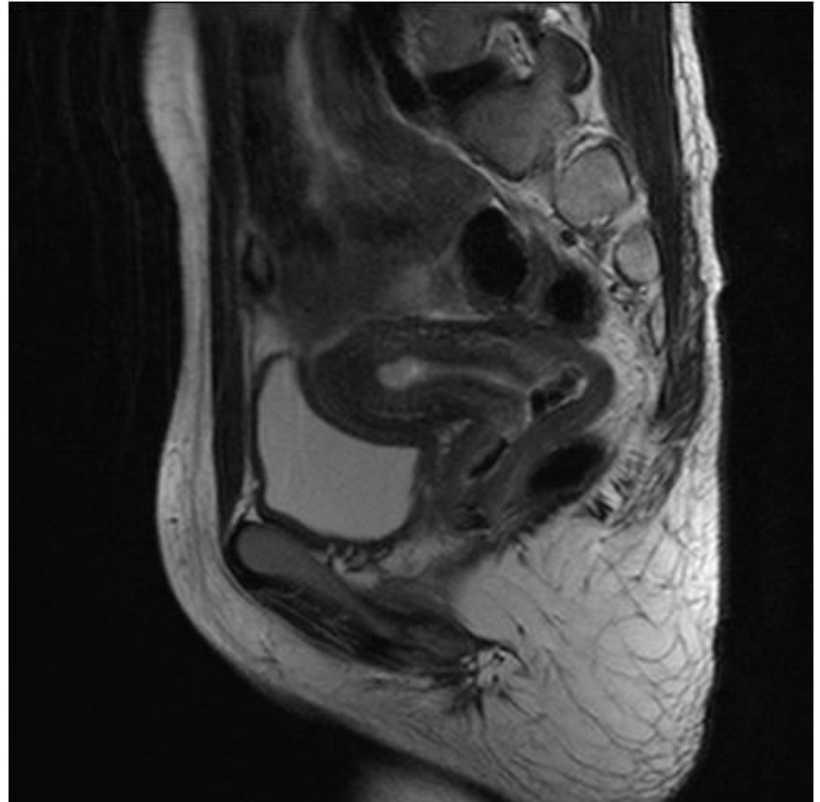

Figure 6: Post operative Magnetic resonance imaging showing complete drainage of hemorrhagic fluid from uterus and vagina along with normal uterus and vagina.

duct and the lower portion is derived from the sinovaginal bulbs. ${ }^{2}$ A transverse vaginal septum may result from a failure of complete canalization of the primordial vaginal plate or from lack of union, which represents the junction of the urogenital sinus and the paramesonephric duct contribution. The site of the septum and thus obstruction may occur at any point along the vaginal canal. The most common location of the transverse septum is the upper vagina (46\%), followed by the middle (35\%), with the least common location being the lower third $(13 \%))^{3}$ The thickness of the septum varies and the one located near the cervix is thicker. The average thickness of a septum is $1 \mathrm{~cm}$ but septum up to 5$6 \mathrm{~cm}$ has also been reported in the literature. ${ }^{3}$ The transverse vaginal septum may be either perforate (incomplete) or imperforate (complete). Unlike other mullerian duct defects, transverse vaginal septum generally is not associated with urologic abnormalities. Our patient had four $\mathrm{cm}$ thick complete low transverse vaginal septum, which is least common. It was associated with low anorectal anomaly, for which she underwent surgical correction at 18 years of age.

The diagnosis of this condition in infancy is difficult as there is no bulging of the outlet. Our case supports this view. In some cases however, the infant may develop hydromucocolpos or hydrometrocolpos and may present with an abdominopelvic mass. It is not unusual to observe dysuria or urinary retention because of urinary tract obstruction associated with the large mucocolpos. ${ }^{4}$

Like in our case, the onset of symptoms usually in case of complete septum occurs after puberty, with the onset of menstruation, when the girl presents with cyclical 
abdominal pain and/or primary amenorrhea. Pelvic pain and pressure are primarily due to the large hematocolpos caused by obstruction to the outflow of menstrual blood because of transverse vaginal septum. The delay in diagnosis may be considerable, approaching 2-3 years. Patients with low vaginal septum present late than the high septum. ${ }^{3}$ However because of typical symptomatology of cyclical abdominal pain along with blind \& non bulging vaginal outlet, we could detect our case early. Incomplete (perforated) septum may manifest later in life, with dyspareunia and dysmenorrhoea, as it permits egress of menstrual blood thro' the opening in the septum.

Diagnosis may be confirmed either by sonography or magnetic resonance imaging (MRI). MRI is superior in assessment of the septal thickness and depth, prior to surgery. Also, MRI is useful in identification of the cervix which is crucial for differentiating a high transverse vaginal septum from congenital absence of the cervix. MRI also helps in easily identifying associated additional anomalies and is critical in helping delineate complex anomalies with marked secondary distortion of the uterovaginal anatomy. ${ }^{5}$ MRI in our case not only helped in the diagnosis and assessment of the septum but was also useful in delineating pelvic structures especially in view of her history of surgery for low anorectal anomaly.

In cases of the very low transverse vaginal septum, there is a possibility of a clinical misdiagnosis of an imperforate hymen. The hymen is the embryologic septum between the sino-vaginal bulbs above and the urogenital sinuses below, and its incidence approximates 1 in 1000 to 2000 females6. Imperforate hymen (bluish purple) bulges out thro introitus, unlike vaginal septum. Moreover, after excision, histology will show the presence of mullerian duct (mesodermal origin) tissue in the septum. ${ }^{7}$

Higher subsequent pregnancy success rate has been reported with repair of a vaginal obstruction due to an imperforate hymen as compared with a complete transverse vaginal septum. Furthermore, pregnancy success is increased in patients with a complete transverse vaginal septum of the lower third of the vagina as compared with those with a high septum. ${ }^{3}$ This is since in patients with a high vaginal septum, retrograde menstruation occurs early after the onset of menstruation as only a small portion of vagina is present that predisposes these patients to the development of endometriosis leading to infertility in these cases. Thus, prompt diagnosis and surgical correction to drain accumulated blood may preserve reproductive capacity by preventing this sequela.

Thin septa may be removed by excision followed by end to end anastomosis of the upper and lower end of the vagina. However in cases of thick vaginal septa, like one in our case, where end to end anastomosis is difficult, skin grafts or amnion graft may be required to cover the defect left by excision the septa. We used amnion graft for this purpose with good result. Garcia reported a Zplasty technique as an alternative to excision with end to end anastomosis, which minimizes the scar formation8. In view of high rate of endometriosis due to retrograde menstruation resulting from outflow tract obstruction, Sanfilippo recommends for concurrent laparoscopy with vaginal septum excision. $^{9}$

\section{CONCLUSIONS}

Transverse vaginal septum is a rare congenital anomaly, leading to uterovaginal outflow tract obstruction. Often, the anomaly is undetected until adolescence, when primary amenorrhea or cyclic, pelvic pain and a suprapubic tender mass prompt a diagnostic evaluation. Ultrasound aids in localization and characterization of the lesion while MRI helps in confirming the vaginal septum including its thickness and relation to the adjacent structures. Timely diagnosis and institution of appropriate management is essential for prevention of short term and long term complications e.g. dysmenorrhoea, dyspaerunia, endometriosis and infertility.

Funding: No funding sources

Conflict of interest: None declared

Ethical approval: Not Required

\section{REFERENCES}

1. Banerjee R, Laufer MR. Reproductive disorders associated with pelvic pain. Semin Pediatr Surg. 1998; 7:52-61.

2. Vishram Singh, Genital system. In: Textbbok of clinical Embryology. 1st edition. Elsevier. 2012:24764.

3. Rock JA, Zacur HA, Dlugi AM. Pregnancy success following surgical correction of imperforate hymen and complete transverse vaginal septum. Obstet Gynecol. 1982;59(4):448.

4. Kathleen Graziano, Daniel H. Teitelbaum, Ronald B. Hirschl. Vaginal Reconstruction for Ambiguous Genitalia and Congenital Absence of the Vagina: A 27-Year Experience: J Pediatr Surg. 2002;37:955-60.

5. Troiano RN, McCarthy SM. Mullerian Duct Anomalies: Imaging and Clinical Issues. Radiology. 2004;233(1):19-34.

6. Parazzini F, Cecchetti G. The frequency of imperforate hymen in northern Italy to diethylstilbestrol. Am J Epidemiol. 1990;19(3):763.

7. Wang J, Ezzat W, Davidson M. Transverse vaginal septum: A case report. The Journal of Reproductive Medicine. 1995;40(2):163-6.

8. Garcia RF. Z-plasty for correction of congenital transverse vaginal septum. Am Obstet gynecol. 1938;141:910.

9. Sanfilippo JS, Wakim NG, Schikler KN. Endometriosis in association with uterine anomaly. Am J Obstet Gynecol. 1986;154(1):39.

Cite this article as: Singh S, Biswas M, Dey M, Nambula V, Abbas F. A rare case of low transverse vaginal septum. Int J Reprod Contracept Obstet Gynecol 2015;4:2103-6. 